\title{
Binary Particle Swarm Optimization Algorithm
}

\section{For Kidney Exchanges}

\author{
Wael M. F. Abdel-Rehim \\ Faculty of Computers and Information \\ Suez University, Egypt \\ wael_fawaz@hotmail.com
}

\begin{abstract}
In this paper, we implement a Particle Swarm Optimization (PSO) based method for solving the kidney exchange problem. Because using a kidney exchange, we can help incompatible patient-donor couples to swap donors to receive a compatible kidney. Implementing Particle Swarm Optimization in solving the kidney exchange problem is an effective method because the obtained results indicate that PSO outperforms other stochastic-based methods such as Genetic Algorithm regarding the efficient the number of resulting exchanges.
\end{abstract}

Keywords - kidney exchange, Particle Swarm, Metaheuristics, Optimization.

\section{INTRODUCTION}

Kidney exchange is one of the few approaches that may always cure people with kidney failure. Moreover, it makes the previously not allowed surgery of incompatible patientdonor pairs easier to be achieved. From the mathematical view, kidney exchange is an optimization problem for the number of possible exchanges among the mismatched pairs in a given pool. Moreover, optimization modelling should consider the expected quality-adjusted life of transplant candidates and operational hospital resources.

There is an opportunity that a patient finds a willing living donor. Still, that patient is not compatible with the donor because of the different blood or tissue kind (incompatible donor-patient pair) [1]. Maybe another couple that has the same problem. Suppose the second couple's donor is compatible with the patient of the first couple and vice versa. In that case, we can create a kidney exchange program, also called kidney paired donation (KPD), that matches incompatible patient-donor couples [2].

Hence, to maximize the number of possible matches between the opposite pairs in a given kidney exchange pool is the goal of a kidney exchange program.

Kidney exchange program could be applied in a static or dynamic environment [1]. In the static context, kidney exchange attempts to find possible matches located in the current pool. However, in the dynamic environment, pool size periodically changes. Because existing pairs can leave, new pairs can arrive, or the patient successfully receives a kidney.
In $[1,3]$ they used the genetic algorithm (GA) with different signified solutions. The genetic algorithm is used to search for the best matches in a specified kidney paired donation pool. However, the genetic algorithm searching method surfers from the optimal local solution. Moreover, the implemented methods are not flexible to be practical in a dynamic environment [1]. Ant Lion Optimization algorithm (ALO) methods reach good results regarding the number of resulting transplants compared to the best-known methods. However, ALO methods did not get a good running time like integer programming [4].

Kennedy and Eberhart [5] initially proposed the Particle Swarm Optimization algorithm for solving optimization problems. Later, it has been successfully applied in many research and application areas.

In this paper, we present an efficient meta-heuristic algorithm for kidney exchanges. Particle swarm optimization algorithm is proposed to find the probable matches in a kidney exchange pool optimally. Particle Swarm Optimization in solving the kidney exchange problem is an evolutionary computation technique that is computationally less expensive and can converge faster than other methods. Moreover, our proposed method can be adapted for solving the kidney exchange in dynamic environments.

\section{THE KIDNEY EXCHANGE PROTOCOLS}

Kidney transplantation is the hope for curing patients with end-stage renal disease. However, The increasing number of waiting patients on the scheduled list for transplantation could disruption the hope of saving more lives. Therefore, a program for kidney exchange is called recently kidney paired donation program [6].

People that deal with the end-stage renal disease can potentially be cured with a kidney transplant. There are two ways for kidneys transplantation. The first way is receiving a kidney from a deceased donor. The other way for kidneys transplantation is to receive a kidney from a willing living donor, which has increased [7]. Moreover, transplanting kidneys from living donors have a high success rate than dead donors [8]. 
Managing KPD programs lies in choosing the "optimal" set of kidney exchanges among several possible alternatives. A typical tree search algorithm is used as one of the first starting solutions to kidney exchange problem.

However, it has a memory space restriction when the patient-donor pairs' size increased [9].

Other researchers solve the kidney exchange problem by formalizing it as an optimization problem. Integer programming method is used to find the optimal matches among incompatible pairs. The goal of the optimization method is to maximize the number of transplants.

The two crucial factors that play a vital role in kidney matching choices are ABO blood kinds and Human Leukocyte Antigen (HLA) tissue. HLA tissue consists of six proteins needed to match the donors and patients to transplant a kidney successfully. There are four blood kinds a patient could have $\mathrm{A}, \mathrm{B}, \mathrm{AB}$, or $\mathrm{O}$. Table 1 shows the matching compatibility rules among the patients and donors according to their blood kinds.

\section{TABLE 1. COMPATIBILITY RULES BASED ON ABO BLOOD KIND}

\begin{tabular}{|c|c|c|c|c|}
\hline Donor/Patient & A & B & AB & O \\
\hline $\mathrm{A}$ & $\mathrm{X}$ & & $\mathrm{X}$ & \\
\hline $\mathrm{B}$ & & $\mathrm{X}$ & $\mathrm{X}$ & \\
\hline $\mathrm{AB}$ & & & $\mathrm{X}$ & \\
\hline $\mathrm{O}$ & $\mathrm{X}$ & $\mathrm{X}$ & $\mathrm{X}$ & $\mathrm{X}$ \\
\hline
\end{tabular}

To understand the kidney exchange. We can represent a kidney paired donation program as a directed graph. Figure 1 shows one example of a kidney exchange. Any number denotes a patient-donor couple that is incompatible with each other. Moreover, the figure represents a two-way exchange cycle.

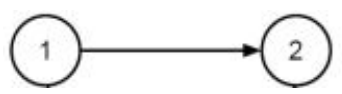

Fig. 1. Kidney exchange with a cycle of two couples

Figure 2 shows another example of a kidney exchange. Every patient from these three couples obtains a kidney, and every donor donates a kidney. Moreover, the figure represents a three-way exchange cycle.

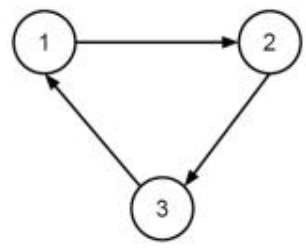

Fig. 2. Kidney exchange by a cycle of three couples

The goal of any kidney exchange program is to find travel in the graph that includes every edge exactly once and gives shared benefits.

The donor who is willing to help and offer their kidney is called altruists, and they do not need a kidney. An example of a kidney exchange by an altruist is shown in figure 3 The kidney exchange begins with an altruistic donor is called a chain

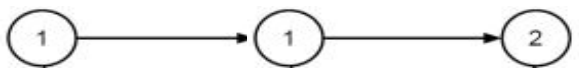

Fig. 3. Kidney exchange with a chain starts with an altruist and two couples

When Kidney paired donation program was performed, the hospitals arranged the exchange locally among their patients. When the number of patients was increased, the availability of kidney exchange programs was universally transformed and controlled by national and international centres.

\section{A. Particle Swarm Optimization: an overview}

Particle Swarm Optimization (PSO) is based on observations of animals' social behaviour, such as birds in flocks or fish in schools. It is an essential evolutionary technique suggested by Kennedy and Eberhart (1995) [5] for optimizing continuous non-linear functions. Later, it has been extended to solve discrete or combinational optimization problems, including scheduling problems and other problems.

PSO is beneficial in a wide diversity of applications. A survey by Poli (2007) [10] reported on the rapid growth in applications and identified hundreds of documented works on PSO.

\section{Standard PSO Algorithm}

Particle swarm optimization is an evolutionary computation technique, fast and straightforward method of optimization. It mimics the behaviours of flocks of birds or fish in schools. PSO is inexpensive regarding both memory requirements and time.

The algorithm's basic concept is that; PSO is initialized with a population from random particles (solutions) and then searches for the fittest solutions to update the population's members. In every iteration, each particle moves in the problem space and has the best value (fitness) that has been achieved (called pbest). The best other matter is the best value achieved by any particle in the population (called gbest). PSO has a velocity that would change the position from the particle. At each iteration, the value of velocity and position are updated. Later finding the two best values, the particle updates its velocity and positions with the following equations (1) and (2).

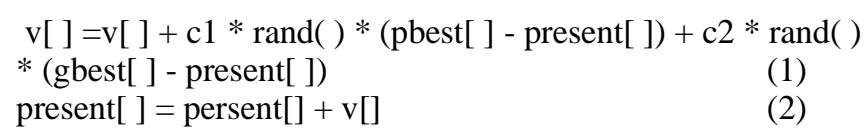

$\mathrm{v}[\mathrm{]}$ is the particle velocity, and persent[] is the present particle (solution). pbest[] and gbest[] are defined as 
specified before. rand () is a random numeral between $(0,1)$. $\mathrm{c} 1, \mathrm{c} 2$ are learning factors, regularly $\mathrm{c} 1=\mathrm{c} 2=2$.

Figure 4 shows how a general PSO algorithm works.

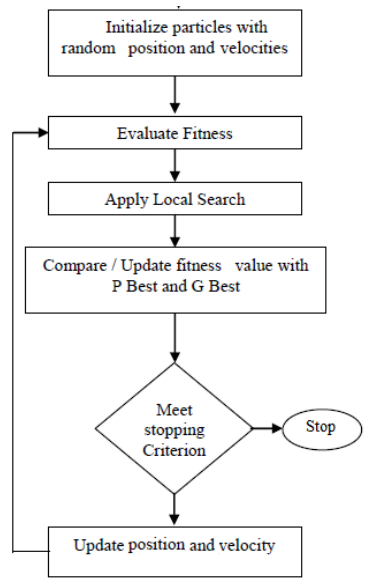

Fig. 4. Particle Swarm Optimization Flow Chart

\section{PSO for solving the kidney exchange problem}

Particle Swarm Optimization algorithm can be adapted to solve the kidney exchange problem. We must also adjust the PSO position representation, particle velocity, and particle movement to work well with combinational optimization problems.

The goal of our proposed method is to maximize the exchange utility while satisfying the problem constraints. Moreover, feasible exchanges are only acceptable (i.e., nodes involved in a specified exchange cannot be used in any further exchanges).

\section{The proposed PSO algorithm for kidney exchanges}

In our proposed PSO approach kidney exchange is formulated as a combinatorial optimization problem. The goal of our optimization problem is to maximize the exchange utility while satisfactory the problem constraints. Feasible exchanges are only permitted. Moreover, the length of the transaction is restricted to the maximum permissible size for chains and cycles.

The proposed method comprises three main stages: First, building the KPD pool using the candidate pairs, the altruistic donors, and the compatible relations. Second, extracting all possible cycles and chains in the existing KPD pool given that the maximum permitted length is k. Finally, providing all of the potential matches to the particle swarm optimization to select the best exchanges or matches maximizes the number of patients receiving a kidney. After each successful kidney transplants, the KPD pool is updated and ready for a new kidney exchange round.

\section{Binary Particle Swarm Optimization}

In our proposed method, each distinct is denoted by a vector with a dimension equal to the total number of exchanges taken out from the specified KPD pool. Usually, individuals of the PSO algorithm are represented by continuous-valued vectors.

However, due to the discrete solution spaces of kidney exchange optimization problems, there are crucial issues in applying PSO successfully to kidney exchanges and finding a suitable mapping between problem solution and PSO particle.

In our proposed method, each individual is denoted by a vector with a dimension equals to the total number of exchanges pull out from the given kidney exchange pool. Moreover, since We formulate the KPD problem as a combinatorial optimization problem, vector values are restricted to the range $[0,1]$.

Several binarization approaches have been proposed to adapt continuous meta-heuristic optimization algorithms for solving binary problems [11].

To extend the PSO algorithm, Kennedy and Eberhart [12] developed a binary particle swarm optimization to solve discrete problems.

Binary PSO is initialized with a population of particles. All particles pass in a problem space to find the optimal solution for each of the iteration. A particle presents a possible solution in an n-dimensional space. Each particle has its position and velocity vectors for controlling its movement. The position and velocity vectors of the $\mathrm{i}^{\text {th }}$ particle in the $\mathrm{n}$ dimension can be denoted as $\mathrm{X}_{\mathrm{i}}=\left(\mathrm{x}_{\mathrm{i}}{ }^{1}, \mathrm{x}_{\mathrm{i}}{ }^{2}, \ldots, \mathrm{x}_{\mathrm{i}}{ }^{\mathrm{n}}\right)$ and $\mathrm{V}_{\mathrm{i}}$ $=\left(\mathrm{v}_{\mathrm{i}}{ }^{1}, \mathrm{v}_{\mathrm{i}}{ }^{2}, \ldots, \mathrm{v}_{\mathrm{i}^{\mathrm{n}}}\right)$, respectively, where $\mathrm{x}_{\mathrm{i}}{ }^{\mathrm{d}} \in\{0$, $1\} ; \mathrm{i}=1,2, . \mathrm{m}$ ( $\mathrm{m}$ is the total number of particles) and $\mathrm{d}=1,2, . . \mathrm{n}$ ( $\mathrm{n}$ is the dimension of data) [12].

Where the velocity vector equation and the position vector equation are defined as velocity vector equation:

$$
V_{i}^{n}(t+1)=\frac{1}{1+e^{-v_{i}^{n}(t)}}
$$

position vector equation:

$$
x_{i}^{n}(t+1)= \begin{cases}1 & \text { if } \mathrm{r}<V_{i}^{n} \\ 0 & \text { otherwise }\end{cases}
$$

Moreover, $\mathrm{r}$ is the random number selected from a uniform distribution in $[0,1]$, enabling the algorithm to output binary positions, not continuous values as seen in global-best or local-best PSO.

The significant difference between PSO and binary PSO is that, in binary PSO, the particle's velocity is updated concerning the particle's probability to be 0 or 1 [13]. 
The goal of the utilized binary PSO algorithm is to maximize the kidney exchange's utility by searching for multiple generations until it finds an appropriate cycle and chain solution.

Fitness function $\mathrm{F}$ is proposed to map each search space into a real-valued $\mathrm{f}$, which is used to evaluate an individual's strength in a given problem.

The proposed fitness function is considered according to the following conditions:

- The Altruistic node means a kidney without taking any benefits in return.

- In the resulting exchanges, the node's donor is used more than once a penalty value is added.

- In the resulting solution, every incompatible pairs node requirement be involved in the kidney exchange only once:

- In the resulting exchanges, the node's receiver is used more than once a penalty value is added.

The proposed fitness function is well-defined by equation (5):

$$
\begin{gathered}
\operatorname{maximize}\left(\sum^{L} \sum^{U} W_{i, j}\right)-E \\
E=\left(E_{1}+E_{2}\right) \lambda
\end{gathered}
$$

where:

- L means the solution length (number of exchanges, i.e., chains or cycles represented by the solution);

- $U$ indicates the exchange length (number of compatible relations represented by the cycle or chain);

- i denotes the node's donor index;

- $\mathrm{j}$ denotes the node's patient index;

- $\mathrm{w}_{\mathrm{i}, \mathrm{j}}$ indicates a utility value for the compatible relation among donor $\mathrm{i}$ and patient $\mathrm{j}$.

- $E_{1}$ means the number of donors which used more than once for donating a kidney;

- $E_{2}$ means the number of patients used more than once that for receiving a kidney;

- $\lambda$ is a penalty value added to maximize error value for the solutions, containing nodes used more than once, $\lambda$ pool size.

A list of mismatched pairs nodes $\mathrm{n}$, and altruistic donors nodes $\mathrm{A}$, the compatibility matrix $\mathrm{V}$. figure 5 shows our proposed algorithm's steps.

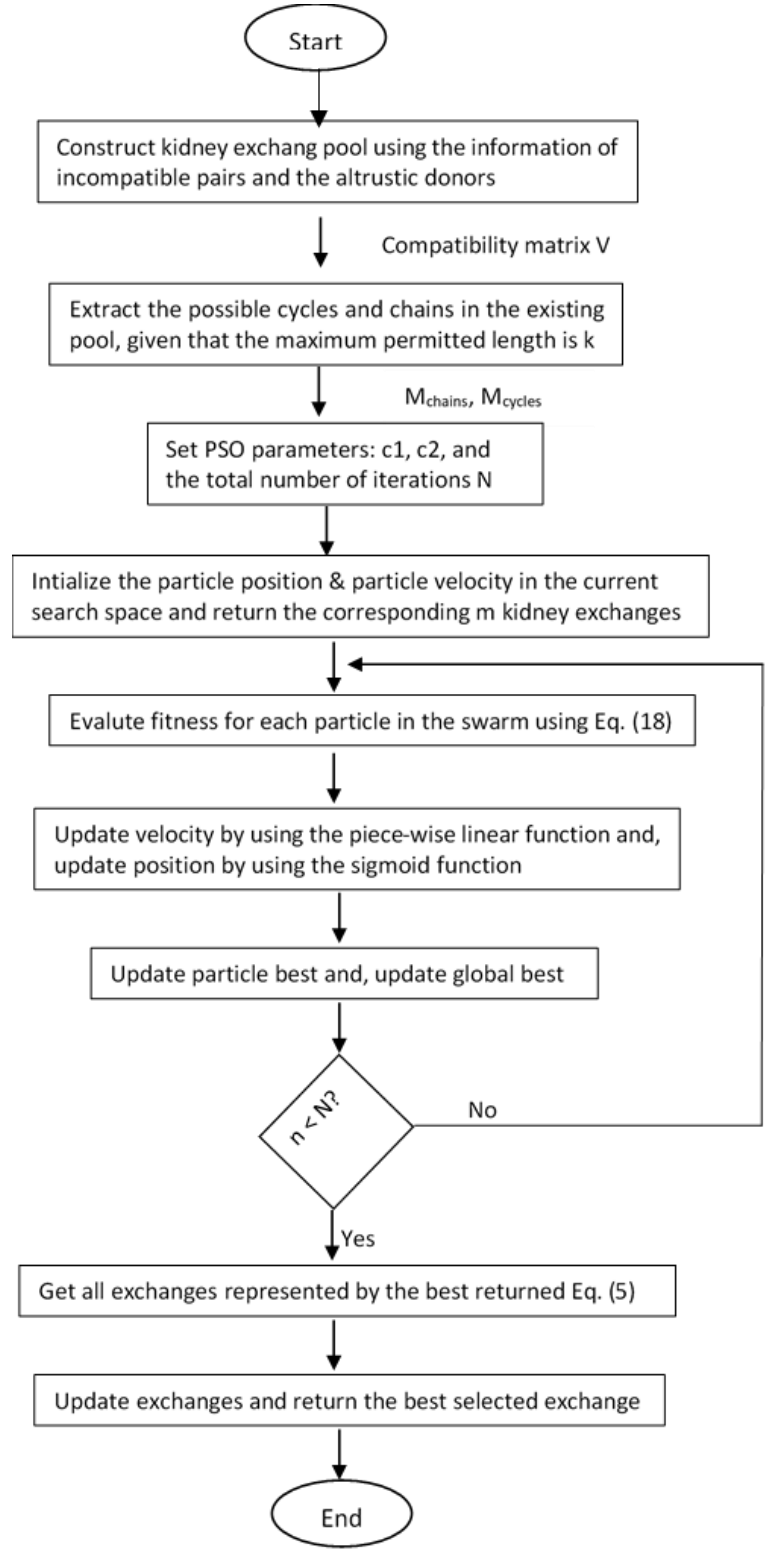

Fig. 5. The proposed ALO algorithm flow chart of for kidney exchanges.

\section{EXPERIMENTAL RESULTS}

In this section, we implement the PSO to solve the kidney exchange problem with MATLAB. The experimental results are stated on Laptop Core i5 $2.50 \mathrm{GHz}$ CPU, 4GB of RAM, and, MATLAB 8.1.

The program starts with a random initial swarm. Then, particles seek their Fitness by implementing the objective function. Since calculating the Fitness, the position, and the velocity of one particle is independent of the other particles.

Our method was evaluated; it is performed using six simulated datasets generated from an updated version of Saidman generator [14]. The PSO starts with using the Saidman generator to create a predefined number of patientdonor couples. The simulated kidney exchange pools have 
some donor-patient pairs changing from 30 to 200 nodes, and the ratio of altruistic donors is selected according to the value described by Saidman generator.

Note that: for every random generation of a kidney pool, the compatibility matching rules encoded in the simulated datasets differ according to it.

We compared our method to Genetic Algorithm and ALO, which are the two known stochastic-based optimization algorithm practical to the kidney exchange space $[1,3,4]$. GA was applied to search for the maximum amount of feasible exchanges in the extracted chains and cycles. Mutation and crossover probabilities for GA were set to 0.2 and 0.8 correspondingly; the used selection method was Roulette Wheel [4]. For easiness, this method is mentioned as GA-KPD. Moreover, we compared our results with the Ant Lion optimization algorithm. We will call this method ALO-KPD (For more details, see [4]).

Moreover, compared with a deterministic-based algorithm for kidney exchanges, Integer Programming (IP) [15] is used to find the optimal exchanges in the obtained cycles and chains. This method will call IP-KPD, and the equation describes its problem formulation:

$$
\begin{aligned}
\text { Maximize } & \sum_{c \in M} \sum^{U} x_{c w_{i, j}} \\
& \text { s.t. } x_{c} \in\{0,1\}, \forall c \in M \\
& \sum_{c \in M(t)} x_{c} \leq 1, \forall t \in \text { Pool }
\end{aligned}
$$

$\mathrm{M}$ is the set of all obtained cycles and chains from KPD pool up to the maximum length equals three. $M(t)$ is a cycle or chain in $M$ that contains node $t$, which represents an altruistic donor-patient or an altruistic pair. $\mathrm{w}_{\mathrm{i}, \mathrm{j}}$ denotes an efficacy value for the compatible relation among donor $i$ and patient $\mathrm{j}$, and $\mathrm{x}_{\mathrm{c}}$ is a binary vector representative whether the cycle or chain $\mathrm{c}$ is chosen for transplant $\left(\mathrm{x}_{\mathrm{c}}=1\right)$ or not $\left(\mathrm{x}_{\mathrm{c}}=\right.$ $0)$. The present constraints are specified as each node can be used only one in a solution.

Table 2. The average number of exchanges/transplants with the standard deviation, returned by ALO-KPD, GA-KPD and our proposed approach, compared to the exact number of exchanges produced by IP-KPD

\begin{tabular}{|c|c|c|c|c|c|}
\hline \multirow{2}{*}{$\begin{array}{c}\text { Pool } \\
\text { Size }\end{array}$} & \multirow{2}{*}{$\mathbf{M}$} & \multicolumn{4}{|c|}{ \# Transplants } \\
\cline { 3 - 6 } & & GA-KPD & ALO-KPD & Proposed & IP-KPD \\
\hline $\mathrm{n}=30$ & 7 & $3 \pm 0$ & $3 \pm 0$ & $3 \pm 0$ & 3 \\
\hline $\mathrm{n}=40$ & 45 & $9.8 \pm 0.8$ & $10.2 \pm 0.98$ & $10.0 \pm 0.9$ & 11 \\
\hline $\mathrm{n}=50$ & 30 & $6.6 \pm 0.7$ & $7 \pm 0$ & $6.9 \pm 0.4$ & 7 \\
\hline $\mathrm{n}=75$ & 64 & $15.4 \pm 0.98$ & $17.4 \pm 0.92$ & $17.6 \pm 0.9$ & 18 \\
\hline $\mathrm{n}=100$ & 129 & $*$ & $19.8 \pm 1.5$ & $20.7 \pm 4.5$ & 24 \\
\hline $\mathrm{n}=200$ & 607 & $*$ & $50.2 \pm 3.16$ & $51 \pm 5.3$ & 53 \\
\hline
\end{tabular}

Table 2 shows that the proposed method generally offers an average number of transplants larger than GA-KPD. When the number of exchanges was more extensive than 64 , and GA-KPD fails to provide any feasible solution. Moreover, in this case, our average method number of transplants greater than ALO-KPD too. Moreover, our proposed method's maximum number of transplants is very close to IP- kidney paired donation nearly in all cases.

Further, compared the optimal solutions produced by all methods, for example, the composition of a generated and the solution for the pool size equaling 40. The cycles and chains of the solutions created by IP-KPD, our proposed method, ALO-KPD, and GA-KPD are shown in figure 6.

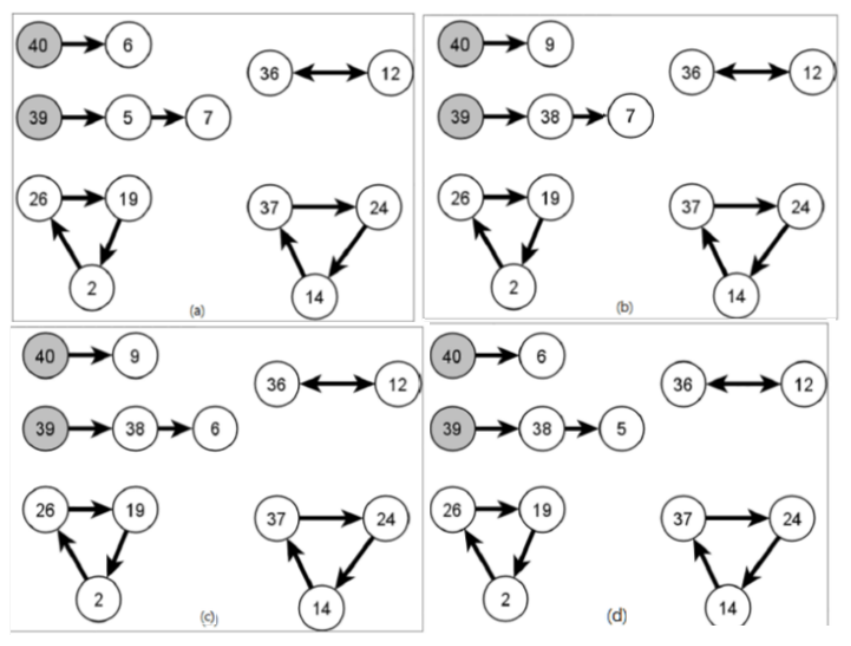

Fig. 6. The composition of an optimal output solution. (a) IPKPD, (b) Proposed, (c) ALO-KPD, (d) GA-KPD.

In the figures, the altruistic node visualizes as a shadedfilled circle where the non-filled circles show the patientdonor pairs' nodes.

The results illustrate that the particle swarm optimization algorithm using MATLAB can effectively solve the kidney exchange problem, especially for large-scale instances. Moreover, our proposed approach is an essential advantage in the flexibility of its application in the kidney exchange in dynamic environments.

\section{CONCLuSion}

In this paper, we have studied the problem of managing a kidney paired donation for saving last stage renal patients' lives. We have implemented the PSO algorithm to solve the kidney exchange problem. Our method searches efficiently for the optimal number of exchange cycles and chains in a specified kidney paired donation pool. Moreover, our approach has comparable exchange results to other competing tools like deterministic Integer Programming.

The results indicate that the PSO algorithm implementation is efficient for solving the kidney exchange problem and other optimization problems.

One of our future insights is implementing our PSO algorithm using parallel MATLAB to reduce the running time for kidney exchange to match patients as soon as possible. 


\section{REFERENCES}

[1] S. Goezinne, R. Bekker, K. Glorie, "A genetic algorithm for kidney transplantation matching", Amsterdam: Business Analytics Master program at VU University, 2016.

[2] A. Roth, T. Sonmez, M. Unver, "Kidney exchange", The Quarterly Journal of Economics, vol. 119, no. 2, pp. 457-488, 2004.

[3] S. Sakthivel, S. Manimaran, "An optimized kidney transplantation based on genetic algorithm", International Journal of Advanced Research, vol. 3, no. 4, 2013.

[4] E. Hamouda, S. El-Metwally, M. Tarek, "Ant Lion Optimization algorithm for kidney exchanges", PLoS ONE, vol. 13, no. 5, 2018

[5] J. Kennedy, R. C. Eberhart, "Particle swarm optimization", Proceedings of the 1995 IEEE International Conference on Neural Networks, Piscataway, New Jersey, pp. 1942-1948, 1995.

[6] D. Ellison, "A Systematic Review of Kidney Paired Donation: Applying Lessons From Historic and Contemporary Case Studies to Improve the US Model", Wharton School, University of Pennsylvania, 2014.

[7] C. Chen, L. Yijiang, D. John, et al., "Graph-Based Optimization Algorithm and Software on Kidney Exchanges", IEEE Transaction on Biomedical Engineering, vol. 59, no. 7, pp. 1985-1991, 2012.

[8] U.S Department of Health. Organ Procurement and Transplantation Network. https://optn.transplant.hrsa.gov. [Accessed Sept. 30, 2018]
[9] D. Abraham, A. Blum, T. Sandholm, "Clearing algorithms for barter exchange markets: enabling nationwide kidney exchange", in the 8th ACM conference on Electronic commerce, California, pp. 295-304, 2017.

[10] R. Poli, "An analysis of publications on particle swarm optimization applications", Essex, UK, Department of Computer Science, University of Essex, 2007.

[11] B. Crawford, et al., "Putting Continuous Metaheuristics to Work in Binary Search Spaces", Complexity, vol. 2017, no. 2, pp.1-19, 2017.

[12] J. Kennedy, R. Eberhart, "A discrete binary version of the particle swarm algorithm. Proc. 1997 IEEE International Conference on Systems, Man, and Cybernetics", IEEE Press, Florida, USA, vol. 8, pp. 4104-4108, 1997.

[13] S. Abid, et al., "Managing Energy in Smart Homes Using Binary Particle Swarm Optimization", In Barolli L., Terzo O. (eds) Complex, Intelligent, and Software Intensive Systems, CISIS 2017, Advances in Intelligent Systems and Computing, vol. 611, Springer, Cham, 2018.

[14] S. Saidman et al., "Increasing the opportunity of live kidney donation by matching for two- and three-way exchanges", Transplantation, vol. 81, no. 5, 2006, pp. 773-782.

[15] A. E. Roth, T. Sönmez, M. U. Ünver, "Efficient kidney exchange: Coincidence of wants in markets with compatibility-based preferences", American Economic Review, vol. 97, no. 3, pp. 828851, 2007. 